\title{
Saturated granular flows: constitutive modelling under steady simple shear conditions
}

\author{
D. VESCOVI*, P. MARVEGGIO* and C. DI PRISCO*
}

\begin{abstract}
In this paper, the authors analyse numerical and experimental results concerning either dry and saturated granular flows under steady, simple shear conditions. A new constitutive model is introduced, on the base of the mixture theory, according to which granular and liquid phases are considered separately. The constitutive relationship refers to the Representative Elementary Volume and assumes the mean values of all kinematic variables, of both granular and liquid phases, to coincide. For the two phases, a parallel scheme is chosen. As far as the granular contribution is concerned, the authors employ an already conceived constitutive model where the critical state concept and the kinetic theory of granular gases are merged, and in which the granular temperature plays the role of state variable for the material. Under saturated conditions, the new model accounts for granular-liquid coupling effects. In fact, the liquid viscosity results to be a function of granular concentration, whereas the evolution of granular temperature is influenced by the liquid molecular viscosity. The model is validated against numerical results and critically discussed. For sufficiently small values of concentration, the transition from Newtonian to Bagnoldian regime, for increasing values of strain rate, is correctly reproduced.
\end{abstract}

KEYWORDS: constitutive relations; theoretical analysis; landslides

\section{INTRODUCTION}

Fast landslides are mass movements of natural materials propagating over long distances. They involve mixtures of fine and coarse grains under saturated and/or unsaturated conditions. At the inception, very often, the soil behaves like a solid (quasi-static conditions), whereas, during propagation, part of the material flows like a granular mixture (dynamic conditions), strains stop localizing and the system is dramatically agitated. In contrast, in case of flow-slides, fluidization takes place at the beginning of flowing and transition from fluid-like to solid-like conditions occurs only when the soil mass stops propagating.

In the context of granular materials, the problem of both defining unified stress-strain relationships under any flow conditions, and reproducing the transition of a solid-like granular mass into a fluid-like flow, and vice versa, seems to be still unsolved. Commonly, the propagation phase has been largely investigated by employing theoretical/numerical tools, according to which the rheology does not take into consideration the two distinct phases (grains and liquid). These approaches assume the moving mass to be a single-phase incompressible material, characterized by a rate-dependent mechanical behaviour. In contrast, according to the authors, in order to describe both inception and propagation stages of fast landslides, the two-phase nature of the material and

Manuscript received.

${ }^{*}$ Department of Civil and Environmental Engineering, Politecnico di Milano, Milano, 20133, Italy the coupling between the liquid and the grains cannot be disregarded.

In the framework of continuum mechanics, the goal of the theoretical approach discussed in this paper is to describe and to predict the mechanical behaviour of the whole medium, starting from the physics of the grain-grain and grain-water interaction. The constitutive model is based on the definition of a Representative Elementary Volume (REV) for the system, in which boundary effects are disregarded since the medium is assumed to be homogeneous in space. Within the REV, whenever grains interact, each other or with water, part of the energy is dissipated, transferred to heat and then dispersed in the environment. In this perspective, the analysis of steady state conditions allows to focus on the dissipative mechanisms governing the process. In fact, under steady and homogeneous conditions, the stored energy is constant, the diffused energy nullifies and all the energy produced is entirely dissipated.

Under dry conditions, the collective mechanical behaviour of a granular system is governed by the properties of the individual particles and the interactions among them. At the micro-scale level, the particle interactions develop two dissipative mechanisms: enduring contacts among grains which are involved in force chains, and inelastic collisions. When the first mechanism prevails, the material behaves like a solid, whereas when the particles interact only under the form of collisions, the material response can be assimilated to that of a fluid. Under saturated conditions, the presence of water changes the dynamics of the grain-grain interaction, and produces additional dissipations due to grain-water contacts. 
In particular, the grain-grain interaction is damped by the presence of the liquid phase, then the energy dissipation of the granular phase increases since the particle movements are bounded by the liquid. As will be discussed in the following, from the liquid phase point of view the presence of immersed grains produces three effects: (i) the liquid streamlines must deviate from their original path in proximity of the grains, behaving these like obstacles for the flow; (ii) lubrication effect. When two or more particles approach each other, the liquid in the region in between them is squeezed out, and its pressure locally increases. (iii) If the grains are not purely transported by the stream, but their motion deviates from the mean flow, the grain velocity fluctuations generate velocity fluctuations in the liquid, which are associated with localized energy dissipation. All the three effects are present even if the mean velocities of the granular and the liquid phase coincide.

Numerical simulations and experiments are powerful tools to investigate the rheology and the dissipative mechanisms of granular mixtures. In the recent years, several papers have been published concerning experimental (Verdugo \& Ishihara, 1996; Huang et al., 2005; Fall et al., 2010; Boyer et al., 2011; Fernandez et al., 2013; Paredes et al., 2013; Dinkgreve et al., 2015; Lin et al., 2015; Pan et al., 2015; Clavaud et al., 2017) and numerical (Fernandez et al., 2013; Seto et al., 2013; Mari et al., 2015; Ness \& Sun, 2015; Singh et al., 2018) results on saturated granular mixtures. Although these results have improved consistently the current state of knowledge on wet granular behaviours, they present some limitations. In particular, laboratory experiments cannot perfectly guarantee homogeneous conditions within the specimen and the results are affected by boundary conditions. In contrast, in numerical simulations, the liquid phase is not realistically reproduced but only its effect is simulated through the implementation of lubrication forces at the contacts between grains (Kim \& Karrila, 1991; Ball \& Melrose, 1997).

The goal of this paper is to propose a constitutive model capable of simulating the behaviour of a mixture of grains saturated with water, under homogeneous and steady shear conditions. The model is formulated within the context of the two-phase mixture theory and, in order to account for the dissipative mechanisms of grain-grain and grainwater interaction, the energy balance equation for the two phases is used. Under dry conditions, the model resumes the theory proposed in Berzi \& Jenkins (2015), in which a unique formulation allows to continuously describe both the solid- and the fluid-like phases. The dry model is based on a parallel scheme, as was proposed in Berzi et al. (2011); Vescovi et al. (2013); Redaelli et al. (2016), according to which the granular stress tensor is calculated as the sum of a quasistatic and a collisional contribution. The two contributions to the stress tensor represent the two previously mentioned dissipative mechanisms, that is force chains and collisions. The originality of the approach proposed in this paper derives from the extension of the dry model to saturated conditions. This goal is achieved by: (i) considering the additional balance equations for the liquid phase (two-phase mixture theory), (ii) including additional dissipative contributions accounting for the presence of water, and (iii) adopting a suitable definition for the liquid viscosity depending on the granular concentration.

\section{DRY AND SATURATED GRANULAR, STEADY FLOWS}

Experiments and numerical simulations performed to study the mechanical behaviour of granular media under both dry (Babic et al., 1990; Campbell, 2002; Ji \& Shen, 2006, 2008; Hatano, 2008; Otsuki \& Hayakawa, 2009; Chialvo et al., 2012; Vescovi \& Luding, 2016) and saturated steady shear conditions (Huang et al., 2005; Fall et al., 2010; Boyer et al., 2011; Paredes et al., 2013; Dinkgreve et al., 2015; Ness \& Sun, 2015), have shown that granular matters can behave like solids or fluids according to the shear rate and the concentration imposed. In the former case the relationship may be assumed to be strain rate-independent, whereas in the latter one cannot. The presence of force chains distinguishes solid-like from fluid-like mechanical responses. Force chains are related to the geometric arrangement of contacts, which can be characterized by means of the fabric tensor. In particular, under homogeneous, simple shear conditions, force chains have been observed to arise when the first invariant of the fabric tensor, i.e. the coordination number, is larger than a critical value (Vescovi et al., 2018). Moreover, under steady, simple shear conditions, there is a one-to-one relation between the coordination number and the concentration, $\nu$ (Sun \& Sundaresan, 2011), where, for a granular material composed of identical particles, $\nu$ is defined as the ratio of the the solid volume of the particles over the total volume of the system (dry or saturated). Then, in the case of steady, homogeneous shear flows, the concentration determines whether the granular system is solidlike or fluid-like, with the phase transition occurring at the critical concentration $\nu_{c}$. For sufficiently small values of shear rate, the stresses are rate-independent when the concentration is larger than $\nu_{c}$. Then, a solid-like behaviour may be expected for $\nu>\nu_{c}$, whereas fluid-like behaviours develop when $\nu<\nu_{c} . \nu_{c}$ depends on the internal micro-structure of the system (Sun \& Sundaresan, 2011; Vescovi et al., 2018), the poly-dispersity (Ogarko \& Luding, 2013; Kumar et al., 2014) and the inter-particle friction, $\mu$, (Chialvo et al., 2012). In particular, in their numerical simulations on steady, homogeneous shear flows of dry, mono-dispersed particles, Chialvo et al. (2012) have measured the critical concentration based on the observation that the pressure fluctuations peak at the phase transition. Similarly Sun \& Sundaresan (2011) identified the critical concentration governing the same transition by extrapolation to zero of the relation between the pressure and the concentration. By fitting the results of their numerical simulations Sun \& Sundaresan (2011) obtained the 
following relation:

$$
\nu_{c}=0.582+0.058 e^{-5 \mu}
$$

For the sake of clarity, it is fundamental specifying that $\nu_{c}$ defined in equation (1) is the critical concentration under very specific conditions:

- the granular material is mono-dispersed,

- all the grains are spherical,

- steady, simple shear conditions are imposed (different values for $\nu_{c}$ are obtained in the case of either compression or extension triaxial tests).

In general, under steady state conditions, for a well determined granular material, the concentrations at which the material stops behaving like a solid and starts transforming in a fluid-like material, is varying according to the type of control imposed (Kumar et al., 2014; Luding, 2016), that is on the Lode angle defined in the strain rate space.

To underline the role played by the liquid in affecting the mechanical behaviour of the material on both solidlike and fluid-like responses, in figure 1 the numerical data of Chialvo et al. (2012) and Ness \& Sun (2015) concerning, respectively, dry (a) and saturated (b) granular systems are compared. All their data are obtained by performing Discrete Element Method (DEM) numerical simulations of steady, homogeneous, shearing flows, under constant volume conditions, on an assembly of particles. The data from Chialvo et al. (2012) concern a mono-disperse, dry system of spherical grains $(\mu=0.5)$. The results from Ness \& Sun (2015) are relative to an ideal suspension (i.e., completely saturated granular material) of spherical particles $(\mu=1)$ in a fluid of molecular viscosity $\eta_{0}$. In particular, the introduction of lubrication terms into the interaction forces among particles mimics the presence of the liquid ("pseudosaturated" conditions). Since lubrication forces are present only when particles interact among each other, when the solid concentration is sufficiently low the liquid response cannot be simulated properly. In such conditions, the mixture behaviour is dominated by that of the liquid, as it will be described later, then also its response may be not realistically simulated by this numerical approach. In order to obtain a more refined set of results, one should consider the application of other numerical techniques, such as Lattice Boltzmann Method (LBM) coupled DEM or Computational Fluid Dynamics (CFD) coupled DEM. LBM and CFD simulate the liquid phase dynamics and, together with DEM, could realistically couple liquid and grains, but in the authors knowledge, up to now, these approaches have not yet been employed to investigate steady, simple shear conditions.

In figure 1 , the dimensionless shear stress $\tau^{*}=\tau d / k_{n}$ versus the dimensionless shear rate $\dot{\gamma}^{*}=\dot{\gamma} d \sqrt{\rho_{p} d / k_{n}}$ measured by Chialvo et al. (2012) and Ness \& Sun (2015), are plotted for different values of $\nu$, being $d, \rho_{p}$ and $k_{n}$ diameter, density and contact elastic stiffness of particles, respectively. The contact stiffness is related to the particles Young modulus $E_{p}$ by the following relation: $k_{n}=\pi d E_{p} / 4$ (Ji \& Shen, 2008). Under both dry and pseudo-saturated conditions, at low shear rates, there are two families of curves, distinguished by $\nu_{c}$. For $\nu<\nu_{c}, \tau^{*}$ continuously decreases for decreasing shear rates (fluid-like behaviour), whereas such a dependence of $\tau^{*}$ on $\dot{\gamma}^{*}$ normally reduces and nullifies for $\nu>\nu_{c}$ (solid-like behaviour). For very large values of $\dot{\gamma}^{*}$, in both cases the dependence on the concentration vanishes and all the curves approach an asymptote characterized by a slope of $1 / 2$. In both figures 1(a) and (b), the fluid-like and solid-like behaviours merge at $\dot{\gamma}^{*} \cong 10^{-2}$.

Since the inter-particle friction coefficient $\mu$ adopted in the two sets of simulations does not coincide, $\nu_{c}$ is slightly different. Nevertheless, the solid-fluid transition occurs in a very narrow range of concentration around the critical value, and in figures 1 (a) and (b) the value of $\nu_{c}$ (which, according to equation (1) should be $\nu_{c}=0.587$ and 0.582 , in the two cases, respectively) cannot be quantitatively measured.

An interesting re-interpretation of the data in figure 1 , is reported in figure 2, where the apparent viscosity, that is the ratio $\tau^{*} / \dot{\gamma}^{*}=\tau / \gamma / \sqrt{\rho_{p} d k_{n}}$, is plotted against $\nu$. The three different sets of numerical data in figure 2 are obtained by imposing different values of the (dimensionless) shear rate, which are almost the same in the dry and saturated cases. In both cases, for $\nu<\nu_{c}$, the apparent viscosity increases with shear rate, whereas for $\nu>\nu_{c}$ it decreases. In the context of non-Newtonian fluid mechanics, the two rheologies are commonly called shear thickening and thinning, respectively. Figure 2 clearly highlights the phase transition between fluidlike and solid-like behaviours, occurring at $\nu \approx \nu_{c}$ where all the curves corresponding to different shear rates intersect each other, providing also a quantitative tool to numerically assess $\nu_{c}$. Independently of whether the granular material is either dry or saturated, solid-like behaviour appears if the particles are packed densely enough $\left(\nu>\nu_{c}\right)$ that a network of persistent contacts develops within the medium, resulting in a jammed mechanically stable structure. On the other hand, when the system is so dilute that persistent force chains cannot develop $\left(\nu<\nu_{c}\right)$, the medium is unjammed and reveals a fluid-like response (Vescovi \& Luding, 2016).

Dry and saturated steady, homogeneous, granular flows behave differently only in the fluid-like regime, for $\nu<\nu_{c}$, at small dimensionless shear rates $\left(\dot{\gamma}^{*}<10^{-2}\right)$. In fact, in the solid-like regime, dry and saturated systems seem to exhibit the same rheology (figure 1), that is the liquid phase seems not to affect the granular response. When $\nu<\nu_{c}$ and $\dot{\gamma}^{*}$ is sufficiently small, in the case of dry systems, the shear stress scales quadratically with shear rate $\tau^{*} \sim \dot{\gamma}^{* 2}$ ("Bagnold scaling", Bagnold, 1954, figure 1(a)). Conversely, when the system is saturated, as is evident in figure 1(b), the shear 


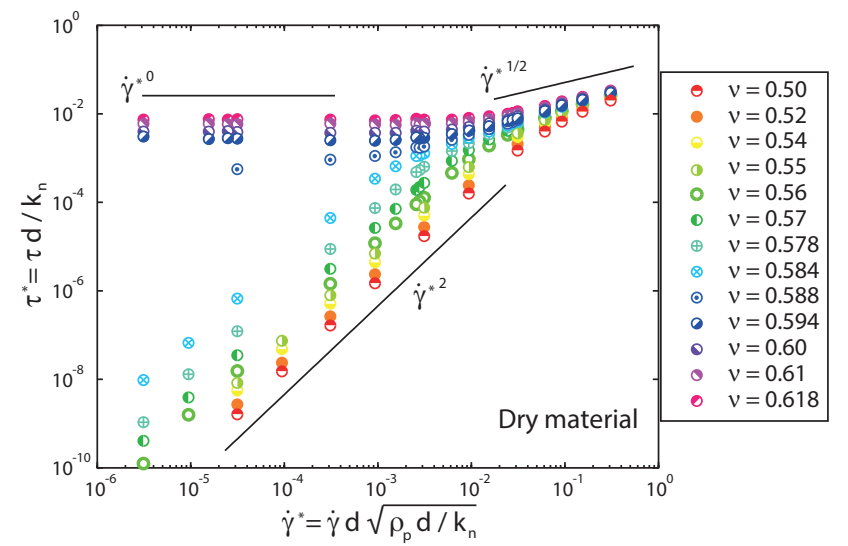

(a)

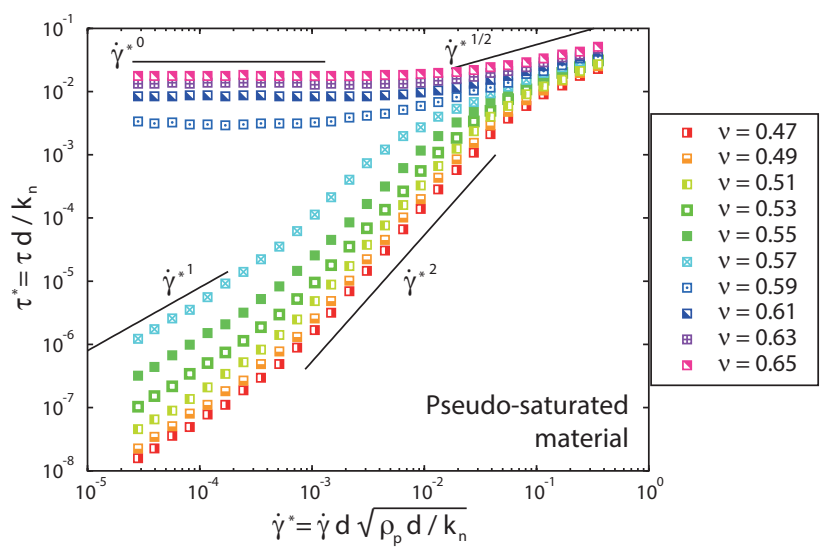

(b)

Fig. 1. Dimensionless shear stress versus dimensionless shear rate for different values of $\nu$, in case of (a) dry granular systems (Chialvo et al., 2012) and (b) non-Brownian suspensions with liquid viscosity $\eta_{0} / \sqrt{\rho_{p} d \dot{\gamma}}=2.15 \cdot 10^{-4}$ (Ness \& Sun, 2015)

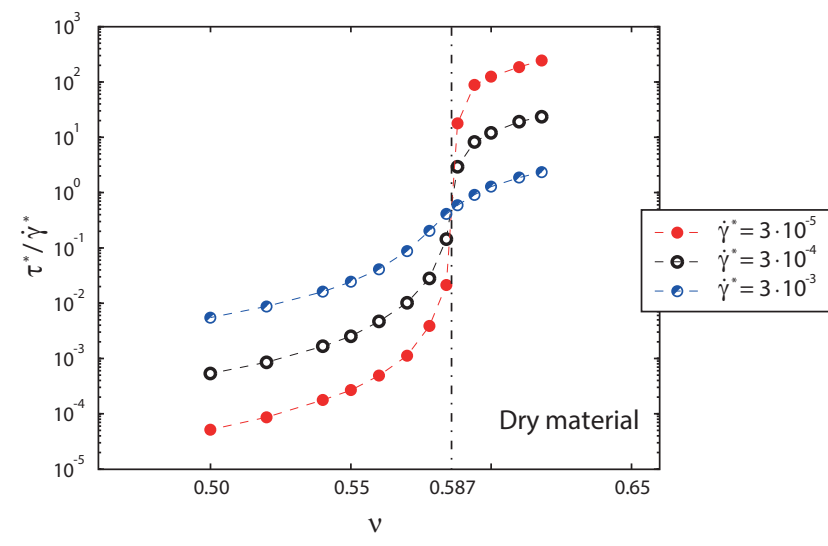

(a)

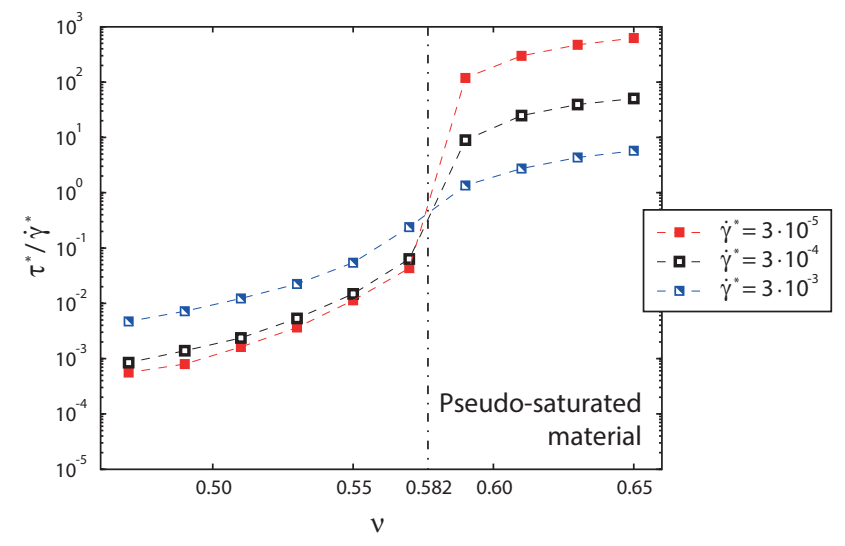

(b)

Fig. 2. Apparent viscosity $\tau^{*} / \dot{\gamma}^{*}=\tau / \dot{\gamma} / \sqrt{\rho_{p} d k_{n}}$ versus $\nu$ for different $\dot{\gamma}^{*}$ values, in case of (a) dry granular systems (by Chialvo et al., 2012) and (b) non-Brownian suspensions with liquid viscosity $\eta_{0} / \sqrt{\rho_{p} d \dot{\gamma}}=2.15 \cdot 10^{-4}$ (by Ness \& Sun, 2015)

stress scales linearly with shear rate $\tau^{*} \sim \dot{\gamma}^{*}$ (Newtonian like regime) at very low shear rates $\left(\dot{\gamma}^{*}<10^{-3}\right)$. In this case, the coefficient/inclination is a function of $\nu$. In contrast, for moderate shear rates $\left(10^{-3}<\dot{\gamma}^{*}<10^{-2}\right), \quad \tau^{*} \sim \dot{\gamma}^{* 2}$ (Bagnoldian like regime). This is emphasized in figures 2(a) and (b): for $\nu<\nu_{c}$, viscosity curves at different shear rates maintain the same separation distance $\left(\dot{\gamma}^{*}\right)$ in the dry case (figure 2(a)), whereas in saturated systems tend to get closer for decreasing shear rates (figure 2(b)).

If the apparent viscosity is plotted against $\dot{\gamma}^{*}$ for $\nu<\nu_{c}$ (figure 3), it becomes apparent that in the saturated system $\tau^{*} / \dot{\gamma}^{*}$ is almost rate-independent for $\dot{\gamma}^{*}<10^{-3}$ (Newtonian regime), and then follows the same trend as in the dry case for $\dot{\gamma}^{*}>10^{-3}$. In the case of dry systems, the apparent viscosity depends linearly on $\dot{\gamma}^{*}$ for $\dot{\gamma}^{*}<10^{-2}$, and for $\dot{\gamma}^{*}>10^{-2}$, continuously decreases until $\tau^{*} / \dot{\gamma}^{*} \sim \dot{\gamma}^{*-1 / 2}$ at large shear rates. In figure 3 , the dry and pseudo-saturated data sets compared are characterized by concentrations which differ similar quantities from the corresponding critical value, $\nu_{c}-\nu \approx 0.03$, then a quantitative comparison is possible.

As a consequence, the influence of the liquid in which the particles are immersed is limited to the Newtonian regime,

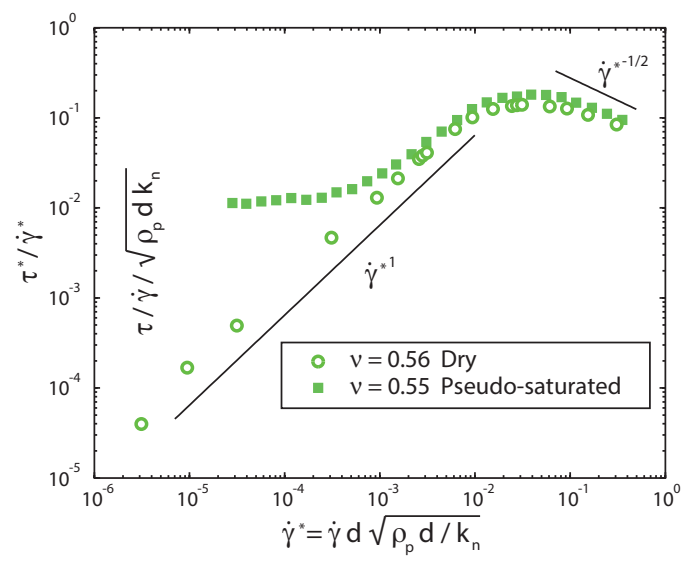

Fig. 3. Comparison of apparent viscosity $\tau^{*} / \dot{\gamma}^{*}=\tau / \dot{\gamma} / \sqrt{\rho_{p} d k_{n}}$ of both dry and saturated granular systems, for $\nu<\nu_{c}$

where $\dot{\gamma}^{*}<10^{-3}$ and $\nu<\nu_{c}$. In this regime, the viscous forces, due to the presence of the liquid, dominate the response of the mixture which coincides with that of a Newtonian liquid. For increasing values of $\dot{\gamma}^{*}$, the particle inertia becomes dominant, and the mixture response is equivalent to that of a dry granular system. It is worth emphasizing that, in the 
Newtonian regime, the response of the mixture is also expected to coincide with that of a pure liquid without suspended grains when the concentration is small. As a consequence, the shear stress should not be affected by the concentration, as for a Newtonian liquid. As noticeable in figure 1(b), this expected behaviour is not simulated by the numerical data. In fact, all the curves maintain the same distance each other in both Bagnoldian and Newtonian regime, then the dependence of $\tau^{*}$ on $\nu$ does not decreases for decreasing values of $\nu$. In the simulations of Ness \& Sun (2015), the liquid phase is not realistically simulated, and the liquid presence is accounted for only by adding lubrication forces at the contacts between particles. This simplification seems to be unable to mimic the mixture rheology in the Newtonian regime, when the system is dominated by the viscous effects due to the liquid phase only.

If the numerical results, discussed here above, are compared with the laboratory experimental test results available in the literature concerning shear flows of saturated mixtures, the theoretical framework becomes further more complex. Experimental tests have been performed by Fall et al. (2010) by using polyethylene spherical particles $\left(\rho_{p}=1050 \mathrm{~kg} / \mathrm{m}^{3}\right.$, $E_{p}=3000 \mathrm{MPa}$ ), of diameter $\mathrm{d}=40 \mu \mathrm{m}$, suspended in water ( $\left.\eta_{0}=10^{-3} \mathrm{~Pa} \cdot \mathrm{s}\right)$. A wide-gap Couette geometry was in this case used to avoid confinement effects. The authors investigated dense suspensions at concentrations below the critical value, identified as $\nu_{c}=0.605$, where the material response is fluidlike. The authors observed the transition from the Newtonian to the Bagnoldian regime in the imposed range of shear rate $\left(\dot{\gamma}=10^{-2} \div 10^{2} \mathrm{~s}^{-1}\right)$. To qualitatively compare numerical (Ness \& Sun, 2015) and experimental (Fall et al., 2010) data, quantities made dimensionless with liquid molecular viscosity, particles diameter and density are here adopted, as is illustrated in figure 4 , where the "relative viscosity", $(\tau / \dot{\gamma}) / \eta_{0}$, is plotted versus the Stokes number, $S t=\dot{\gamma} \rho_{p} d^{2} / \eta_{0}$, for the two data sets. The Stokes number represents the ratio of particle inertia to the viscous forces, and allows to distinguish between Newtonian and Bagnoldian regime. The range of Stokes number adopted in the experiments is 4 orders of magnitude smaller with respect to the simulations. This seems to be due to the fact that, in laboratory tests, apparatus and particles have small dimensions, whereas in simulations large values of $\dot{\gamma}$ are usually imposed to avoid large computational costs. Nevertheless, the two data sets show similar features, scaled by a factor of $10^{-3}$.

According to the authors, the quantitative discrepancies between experimental and numerical data could be ascribed to the dimension of the particles to be employed to define the dimensionless Stokes number. In the plot of figure 4, the length scale of the problem is assumed to be the particle diameter, as each particle behaves independently without forming small clusters. Though, each polyethylene particle is cross-linked to the others forming polymers and therefore cluster formation is expected, although this phenomenon has not been investigated

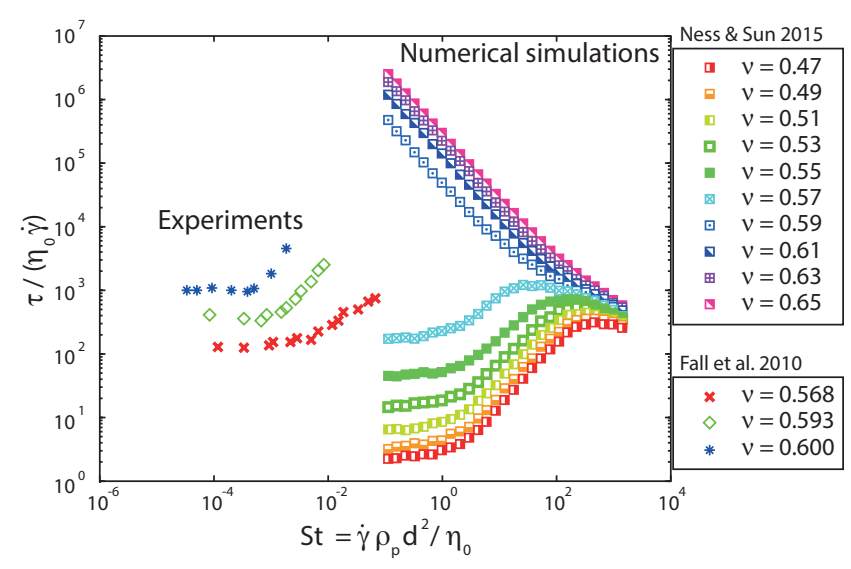

Fig. 4. Comparison between numerical and experimental data in terms of relative viscosity $(\tau / \dot{\gamma}) / \eta_{0}$ versus Stokes number $S t=$ $\dot{\gamma} \rho_{p} d^{2} / \eta_{0}$

in the experiments. Considering for example a cluster having an equivalent diameter ten times greater than the one of the particle, the experimental data set would be scaled by a factor of $10^{2}$, almost overlapping with numerical data.

\section{MODEL THEORETICAL ASSUMPTIONS}

In this paper, a two-phase (grains and liquid) material is considered. For the sake of simplicity, the granular phase is assumed to be an assembly of identical spheres of diameter $d$ and constant density $\rho_{p}$, immersed in a liquid of constant density $\rho_{l}$ (where subscript " $l$ " stands for liquid). The density of the granular phase $\rho_{g}$ can be computed as $\rho_{g}=\rho_{p} \nu$ (where subscript " $g$ " stands for granular).

In order to clarify the rheological approach proposed, the energy balance equation for the mixture is here below taken into consideration. In contrast, the standard mass and momentum balances (Drew, 1983; Jackson, 2001; Pitman \& Le, 2005) are not reported for the sake of brevity, since these latter are not employed in the following.

The energy balance for the mixture can be written as follows:

$$
\begin{aligned}
\boldsymbol{\sigma}: \dot{\varepsilon}= & \rho_{p} \nu\left(\frac{\partial \chi_{g}}{\partial t}+\mathbf{u}_{g} \cdot \nabla \chi_{g}\right)+ \\
& +\rho_{l}(1-\nu)\left(\frac{\partial \chi_{l}}{\partial t}+\mathbf{u}_{l} \cdot \nabla \chi_{l}\right)+ \\
& +\left(\mathbf{u}_{g}-\mathbf{u}_{l}\right) \cdot \mathbf{B}+\nabla \cdot \mathbf{q}+\Gamma
\end{aligned}
$$

where $\sigma$ stands for the total stress tensor and $\dot{\varepsilon}$ for the strain rate tensor, unique for both phases since no mean relative motion is assumed: $\mathbf{u}_{l}=\mathbf{u}_{g}$, where $\mathbf{u}_{g}$ and $\mathbf{u}_{l}$ are the local velocities of the granular and liquid phases, respectively. In equation (2), $\mathbf{q}$ stands for the energy flux, $\mathbf{B}$ is the buoyancy force, $\chi_{g}$ and $\chi_{l}$ are the internal energies and $\Gamma$ is the system dissipated energy. Owing to the assumption of considering steady state, homogeneous and simple shear conditions, equation (2) becomes

$$
\tau \dot{\gamma}=\Gamma
$$


where $\tau$ and $\dot{\gamma}$ are the shear stress and the shear strain rate, respectively. By separating the two contributions (granular and liquid), equation (3) may be written as follows

$$
\left(\tau_{g}+\tau_{l}\right) \dot{\gamma}=\Gamma_{g}+\Gamma_{l}
$$

In particular, $\Gamma_{g}$ accounts for the energy dissipated at the contacts between two or more grains, whereas $\Gamma_{l}$ represents the energy dissipated by the liquid:

$$
\Gamma_{l}=\Gamma_{l}^{E}+\Gamma_{l}^{L}+\Gamma_{l}^{g l},
$$

where: $\Gamma_{l}^{E}$ is the energy dissipated by the liquid due to the presence of the immersed grains which behave as obstacles for the liquid flow (Einstein, 1905); $\Gamma_{l}^{L}$ is the additional dissipation associated with particle collisions. The liquid is squeezed out from the space between the surfaces of the colliding particles and its pressure locally increases. This phenomenon is known in the literature as lubrication effect (Stachowiak \& Batchelor, 2013; Fernandez et al., 2013). Finally, $\Gamma_{l}^{g l}$ is a further contribution due to the grain agitation (fluctuations of grain velocities) within the liquid phase and not directly correlated to collisions.

According to the authors, for the sake of clarity, equations (4)(5) may be written as:

$$
\begin{aligned}
\tau_{g} \dot{\gamma}-\Lambda & =\Gamma_{g}, \\
\tau_{l} \dot{\gamma}+\Lambda & =\Gamma_{l}^{E}+\Gamma_{l}^{L}+\Gamma_{l}^{g l}
\end{aligned}
$$

where $\Lambda$ stands for the energy transferred by the granular to the liquid phase. Equation (6) implies the agitated, fluctuating motion of grains to be damped by the presence of the liquid. In equation (7), $\Lambda$ coincides, by assumption, with $\Gamma_{l}^{g l}$. In other words, the granular phase transfers part of its kinetic energy $(\Lambda)$ to the liquid phase which dissipates it as thermal energy:

$$
\tau_{l} \dot{\gamma}=\Gamma_{l}^{E}+\Gamma_{l}^{L}
$$

Equations (6) and (8) will be employed in the following sections to define the rheologies for both granular and liquid phases.

\section{THE CONSTITUTIVE MODEL}

As is commonly assumed in the geomechanical community, the "total" stress tensor of the granular-liquid mixture, $\sigma$, can be written as the sum of the granular and the liquid contributions:

$$
\boldsymbol{\sigma}=\boldsymbol{\sigma}_{g}+\boldsymbol{\sigma}_{l}
$$

Equation (9) can also be interpreted as a generalization of the effective stresses definition (Terzaghi, 1943), according to which, the stresses attributed to the granular phase are supposed to work in parallel with the liquid contribution $\sigma_{l}$. Differently from the original principle, here the deviatoric liquid contribution is assumed not to be negligible. Analogously, according to the effective stress principle, $\sigma_{g}$ coincides with the stress associated with the arising of force chains, whereas, in the model illustrated hereafter, $\boldsymbol{\sigma}_{g}$ does not nullify even if force chains disappear, that is when $\nu<\nu_{c}$.

According to the assumption of considering simple shear conditions, only the shear and the normal stress components of the stress tensor, $\tau$ and $\sigma$, respectively, have to be defined, that is:

$$
\begin{gathered}
\tau=\tau_{g}+\tau_{l}, \\
\sigma=\sigma_{g}+\sigma_{l} .
\end{gathered}
$$

\section{Granular phase}

To model the granular contribution, the theoretical approach proposed by Berzi \& Jenkins (2015) for dry granular systems is here adopted. This is based on the standard kinetic theory of granular gases (Jenkins \& Savage, 1983; Lun, 1991; Campbell, 1990; Garzó \& Dufty, 1999; Goldhirsch, 2003). In the context of kinetic theories, an additional internal state variable for the granular system is introduced: the granular temperature $T$. The granular temperature represents a measure of the system agitation, and is defined as:

$$
T=\frac{1}{3}\left\langle\left\langle\left|\mathbf{u}_{g}-\mathbf{v}_{p}\right|^{2}\right\rangle\right\rangle
$$

where $\mathbf{v}_{p}$ is the instantaneous single-particle velocity, $\mathbf{u}_{g}$ is the mean velocity of the granular phase defined as $\mathbf{u}_{g}=\left\langle\left\langle\mathbf{v}_{p}\right\rangle\right\rangle$, where $\langle\langle\cdot\rangle\rangle$ designates the average obtained by using the singleparticle velocity distribution function.

The model of Berzi \& Jenkins (2015) is developed as an extension of the standard kinetic theory to account for the deformability of particles, and works according to a "parallel scheme" (Berzi et al., 2011; Vescovi et al., 2013; Redaelli et al., 2016). According to this approach, at the microscale two are the possible dissipative mechanisms due to the interaction among particles: (i) the former one associated with enduring contacts among the grains involved in force chains, and (ii) the latter one with nearly instantaneous inelastic collisions. When the first mechanism prevails, that is when the concentration is sufficiently high, the dry granular material is assumed to behave like a solid (quasi-static regime). On the other hand, when the medium is dilute, a stable contact network cannot develop in the system and particles interact mainly by means of collisions. In this case, the material response can be assimilated to that of a fluid (collisional regime).

The energy dissipation related to the granular phase, $\Gamma_{g}$, is thus assumed to be the sum of two contributions:

$$
\Gamma_{g}=\Gamma_{\mathrm{qs}}+\Gamma_{\mathrm{col}}
$$

where $\Gamma_{\mathrm{qs}}$ is the rate at which energy is dissipated by enduring contacts, and $\Gamma_{\text {col }}$ is the energy dissipated by collisions. Analogously to what already done for the grain-liquid mixture, 
the strain rate tensor is assumed to be unique and the granular stress tensor is split as it follows:

$$
\begin{gathered}
\tau_{g}=\tau_{\mathrm{qs}}+\tau_{\mathrm{col}}, \\
\sigma_{g}=\sigma_{\mathrm{qs}}+\sigma_{\mathrm{col}} .
\end{gathered}
$$

Subscripts " $q s$ " and "col" stand for quasi-static and collisional, respectively. By substituting equations (13)-(14) into equation (6) and by assuming the energy produced by the quasi-static contribution to be completely balanced by the quasi-static dissipated energy ( $\left.\tau_{\mathrm{qs}} \dot{\gamma}=\Gamma_{\mathrm{qs}}\right)$, the energy balance equation for the granular phase reduces to:

$$
\tau_{\mathrm{col}} \dot{\gamma}=\Gamma_{\mathrm{col}}+\Lambda
$$

According to the model, the rate-independent contributions of the stresses are associated with the development of force chains within the medium. Only when a contact network originates in the system, the material behaviour is solidlike, rate-independent and quasi-static stresses arise. This occurs when $\nu>\nu_{c}$; i.e., $\nu_{c}$ is assumed to be the minimum concentration allowing the contact network to develop under steady conditions. In contrast, when $\nu<\nu_{c}$, force chains are inhibited, the material response is fluid-like since particles can interact only by means of collisions, and stresses show a severe rate-dependency.

At the steady state,

$$
\begin{aligned}
\sigma_{\mathrm{qs}} & =F_{0}(\nu) \frac{k_{n}}{d}, \\
\tau_{\mathrm{qs}} & =\sigma_{\mathrm{qs}} \tan \phi_{s s}^{\prime},
\end{aligned}
$$

where $\phi_{s s}^{\prime}$ is the internal friction angle of the material under simple shear conditions. In equation (17), $F_{0}$ is a dimensionless function defined as:

$$
F_{0}(\nu)= \begin{cases}0, & \text { if } \nu \leq \nu_{c} \\ a_{0}\left(\nu-\nu_{c}\right)^{2 / 3}, & \text { if } \nu>\nu_{c}\end{cases}
$$

being $a_{0}$ a dimensionless material parameter. The dependence on $\nu$ is taken from Chialvo et al. (2012).

According to this approach, the critical state (Roscoe et al., 1958; Schofield \& Wroth, 1968; Muir Wood, 1990) is interpreted as the steady state for $\dot{\gamma}$ going to zero. In fact, as it will be described in the following, in the limit of $\dot{\gamma} \rightarrow 0 \sigma_{\text {col }}$ vanishes in equation (15). Then, in such conditions, the granular stress component of the normal stress coincides with the quasistatic one and depends only on the concentration, accordingly to equation (17). Under this condition, $\sigma_{g}$ plays the role of the classical soil mechanics effective normal stress, associated with the solid skeleton contact network (force chains activation). As far as the collisional contribution is concerned, according to
Berzi \& Jenkins (2015):

$$
\begin{aligned}
\sigma_{\mathrm{col}} & =F_{1}(\nu, T) T, \\
\tau_{\mathrm{col}} & =F_{2}(\nu, T) T^{1 / 2} \dot{\gamma}, \\
\Gamma_{\mathrm{col}} & =\frac{F_{3}(\nu, T)}{L(\nu)} \frac{T^{2}}{\dot{\gamma}},
\end{aligned}
$$

where $F_{1}, F_{2}$ and $F_{3}$ are characterized by a similar definition:

$$
F_{j}(\nu, T)= \begin{cases}\frac{f_{j}(\nu) g_{j}(\nu) T^{-1 / 2}}{f_{j}(\nu)+g_{j}(\nu) T^{-1 / 2}}, & \text { if } \nu \leq \nu_{c} \\ g_{j}(\nu) T^{-1 / 2}, & \text { if } \nu>\nu_{c}\end{cases}
$$

with $j=1,2,3$. Function $L$ in equation (22) is named correlation length and accounts for the presence of clusters of particles occurring at sufficiently large concentrations, close to $\nu_{c}$. In particular, for small values of $\nu, L$ tends to $d$ (diameter of grains), whereas for $\nu>\nu_{c}, L$ is constant and equal to its value at $\nu=\nu_{c}$. The expressions for $f_{j}, g_{j}$, for $j=1,2,3$, and $L$ are listed in the Appendix to the paper.

The collisional terms of the constitutive relationship are a function of granular temperature $T$. To evaluate this latter, the collisional part of the granular energy balance equation, equation (16), is employed. In particular, the first term right of equation (16) is a function of $\nu$ and $T$ as in equation (22), whereas $\Lambda$ is a priori unknown. As was previously mentioned, $\Lambda$ is expected to be defined as a function of $T$ since it represents the part of fluctuating energy of grains transferred to the liquid, and there dissipated.

Since the approach introduced assumes:

- a collection of particles immersed in a Newtonian liquid characterized by a molecular viscosity $\eta_{0}$,

- no mean relative motion among the granular and liquid phases $\left(\mathbf{u}_{l}=\mathbf{u}_{g}\right)$,

- inertial effects not to affect the system behaviour (Stokes regime),

then:

$$
\Lambda=\sum_{i=1}^{n} F_{i}^{D}\left|\mathbf{u}_{l}-\mathbf{v}_{p, i}\right|
$$

where

$$
F_{i}^{D}=3 \pi d \eta_{0}\left|\mathbf{u}_{l}-\mathbf{v}_{p, i}\right|
$$

is the Drag force on the $i$-th spherical particle (Stokes, 1851) and $n=6 \nu /\left(\pi d^{3}\right)$ is the number of particles per unit volume. Since $\mathbf{u}_{l}=\mathbf{u}_{g}$, by substituting equation (25) into equation (24), and from the definition of granular temperature, equation (12), it follows:

$$
\Lambda=\frac{54}{d^{2}} \nu \eta_{0} T
$$


By substituting equations (21), (22) and (26) into equation (16), the dependence of $T$ on $\nu$ and $\dot{\gamma}$ is derived:

$$
\frac{F_{3}(\nu, T)}{L(\nu)} T^{2}+\frac{54}{d^{2}} \nu \eta_{0} T \dot{\gamma}-F_{2}(\nu, T) T^{1 / 2} \dot{\gamma}^{3}=0
$$

Equation (27) does not include singularities, but cannot, in general, be solved explicitly for $T$. This derives from the assumption of Stokes regime. As is evident, for $\dot{\gamma}$ going to zero, $T$ nullifies, and, consequently, also $\sigma_{\mathrm{col}}$ and $\tau_{\mathrm{col}}$ vanish.

\section{Liquid phase}

As was previously mentioned, the liquid phase is assumed to be Newtonian and incompressible, so that:

$$
\tau_{l}=\eta(\nu) \dot{\gamma}
$$

being $\eta$ a suitable defined viscosity for the liquid, depending on the concentration, accounting for the dissipation of energy due the presence of the suspended granular phase.

The authors assume:

$$
\eta(\nu)=\eta_{0} F_{\eta}(\nu)
$$

where

$$
F_{\eta}(\nu)= \begin{cases}C_{\eta} \frac{f_{\eta}(\nu)}{f_{\eta}(\nu)+C_{\eta}}, & \text { if } \nu \leq \nu_{c} \\ C_{\eta}, & \text { if } \nu>\nu_{c}\end{cases}
$$

is the non-dimensional viscosity function, whereas $C_{\eta}$ is a nondimensional constitutive parameter and $f_{\eta}$ is a non-dimensional function describing the dependency of $\eta$ on the concentration for sufficiently small values of $\nu$.

In equation (30), $f_{\eta}(\nu)$ and the $C_{\eta}$ value are filleted according to an expression analogous to that used for the collisional terms in equation (23).

For $\nu>\nu_{c}$, force chains develop within the granular phase, therefore the number of grains experiencing collisions may be assumed to be constant with $\nu$. This implies that even the dissipation of energy related to the inertial contacts within the liquid phase gets a constant value, too. It is worth noting that at any rate the quantitative value of $\tau_{l}$, for $\nu>\nu_{c}$, is totally negligible with respect to $\tau_{g}$, which is lower bounded by $\tau_{\mathrm{qs}}$. Therefore, according to equation (30), $F_{\eta}$ gets the constant value $C_{\eta}$ when $\nu>\nu_{c}$.

For $\nu<\nu_{c}$, to define $f_{\eta}(\nu)$ the authors decided to employ the experimental results of Eilers (1941); Roscoe (1952); Krieger \& Dougherty (1959); Chang \& Powell (1994) according to which, if $\dot{\gamma} \rightarrow 0$ and the mixture is interpreted as a onephase material:

$$
\tau=\eta_{0}\left(1-\frac{\nu}{\nu_{c}}\right)^{-2.5 \nu_{c}} \dot{\gamma}
$$

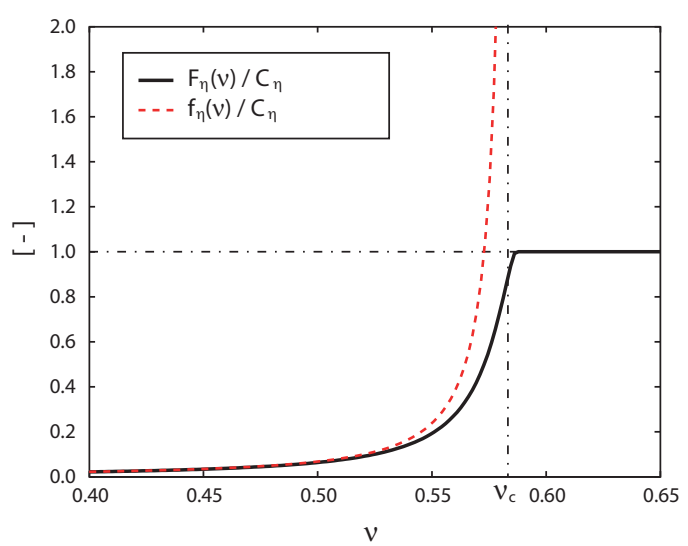

Fig. 5. Non-dimensional viscosity function, $F_{\eta} / C_{\eta}$ (solid line, equation (30)), and $f_{\eta} / C_{\eta}$ (dashed line, equation (35)) versus $\nu$

For $\nu$ values sufficiently small, this relationship asymptotically tends to the well known Einstein (1905) function:

$$
\tau=\eta_{0}(1+2.5 \nu) \dot{\gamma}
$$

According to equations (10) and (14):

$$
\tau=\tau_{\mathrm{col}}+\tau_{\mathrm{qs}}+\tau_{l}
$$

Since the previously mentioned experimental results refer to $\nu<\nu_{c}$ and $\dot{\gamma} \rightarrow 0$, equation (33) reduces to:

$$
\tau=\tau_{l}
$$

in fact the collisional shear stress depends on $\dot{\gamma}$ more than linearly, whereas $\tau_{l} \sim \dot{\gamma}$.

From equations (28)-(31) and (34):

$$
f_{\eta}(\nu)=\left(1-\frac{\nu}{\nu_{c}}\right)^{-2.5 \nu_{c}}
$$

As an example, in figure 5, the complete evolution of $F_{\eta}$ with $\nu$ is plotted.

\section{STEADY SIMPLE SHEAR CONDITIONS \\ Calibration}

The constitutive parameters can be distinguished in micro- and macro-mechanical. In particular, micro-mechanical parameters relative to the granular phase are: particle diameter $d$, density $\rho_{p}$, Young modulus $E_{p}$, coefficient of normal restitution $e_{n}$ and inter-particle friction coefficient $\mu$; whereas macro-mechanical parameters are: the critical concentration $\nu_{c}$, the internal friction angle of the material under simple shear conditions $\phi_{s s}^{\prime}$, and the dimensionless coefficients $a_{0}$ and $C_{\eta}$ appearing in equations (19) and (30), respectively. Solid phase micromechanical parameters and the liquid molecular viscosity $\eta_{0}$ do not require any calibration, while $\nu_{c}$ and $\phi_{s s}^{\prime}$ can be put in relation with the micro-parameters: $\nu_{c}$ is given by equations (1) as a function of $\mu$ and an expression for $\phi_{s s}^{\prime}$ has been provided 
Table 1. List of material parameters. First and second lines: parameter to simulate numerical results of Chialvo et al. (2012) and Ness \& Sun (2015) on dry and pseudo-saturated granular systems, respectively. Third line: parameters simulating the mechanical behaviour of an ideal water saturated soil (critical discussion)

\begin{tabular}{|l|c|c|c|c|c|c|c|}
\hline Material & $e_{n}$ & $\mu$ & $\nu_{c}$ & $\tan \phi_{s s}^{\prime}$ & $a_{0}$ & $\eta_{0} / \sqrt{\rho_{p} d k_{n}}$ & $C_{\eta}$ \\
\hline Dry simulations & 0.7 & 0.5 & 0.587 & 0.386 & 0.20 & - & - \\
Pseudo-saturated simulations & 0.7 & 1 & 0.582 & 0.393 & 0.25 & $2.15 \cdot 10^{-4}$ & 247 \\
Saturated soil & 0.7 & 0.5 & 0.587 & 0.386 & 0.20 & $10^{-6}$ & 247 \\
\hline
\end{tabular}

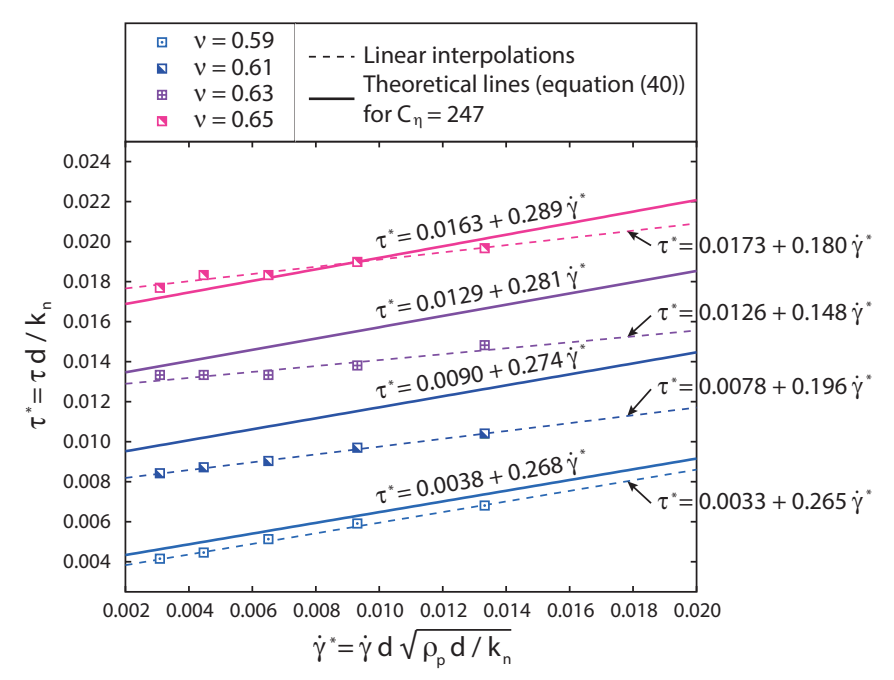

Fig. 6. Calibration of $C_{\eta}$ for $\nu>\nu_{c}$

in Berzi \& Jenkins (2015), as reported in the Appendix. In contrast, the unique parameters to be calibrated are $a_{0}$ and $C_{\eta}$. In this paper, $a_{0}$ has been derived from the numerical data of Chialvo et al. (2012), who performed 3D DEM numerical simulations on granular assemblies of identical spheres with different $\mu$ values. Therefore, here below only the calibration of $C_{\eta}$ is discussed.

To this goal, the authors used the numerical results obtained by Ness \& Sun (2015), for $\nu>\nu_{c}$, reported in figure 6 in terms of $\tau^{*}$ against $\dot{\gamma}^{*}$.

According to equations (21), (23), (28)-(30) and (33), for $\nu \geq$ $\nu_{c}$ :

$$
\tau=\tau_{\mathrm{qs}}+\left[g_{2}(\nu)+C_{\eta} \eta_{0}\right] \dot{\gamma}
$$

and, under non-dimensional form:

$$
\tau^{*}=\tau_{\mathrm{qs}} \frac{d}{k_{n}}+\left[g_{2}(\nu)+C_{\eta} \eta_{0}\right] \frac{1}{\sqrt{\rho_{p} d k_{n}}} \dot{\gamma}^{*} .
$$

In equation (37), from equations (18)-(19) and considering $k_{n}=\pi d E_{p} / 4$,

$$
\tau_{\mathrm{qs}} \frac{d}{k_{n}}=a_{0}\left(\nu-\nu_{c}\right)^{2 / 3} \tan \phi_{s s}^{\prime},
$$

whereas, from the definition of $g_{2}(\nu)$ reported in the Appendix,

$$
\frac{g_{2}(\nu)}{\sqrt{\rho_{p} d k_{n}}}=\frac{2}{3 \sqrt{\pi}} \nu J(\nu)
$$

where $J(\nu)$ is given in the Appendix. Then, equation (37) becomes

$\tau^{*}=a_{0}\left(\nu-\nu_{c}\right)^{2 / 3} \tan \phi_{s s}^{\prime}+\left[\frac{2}{3 \sqrt{\pi}} \nu J(\nu)+C_{\eta} \frac{\eta_{0}}{\sqrt{\rho_{p} d k_{n}}}\right] \dot{\gamma}^{*}$.

By imposing the angular coefficients of equation (40) to coincide with the angular coefficients of the interpolating straight lines of figure 6 (dashed lines), and by averaging over the values obtained for the different $\nu$, it results $C_{\eta}=247$. The solid lines in figure 6 represent equation (40) where $C_{\eta}=247$ and the micro- and macro-parameters are those corresponding to the simulations of Ness \& Sun (2015), as listed in Table 1.

\section{Validation}

The dry, theoretical model has been validated against numerical results of Ji \& Shen (2006), Chialvo et al. (2012) and Chialvo \& Sundaresan (2013) in Berzi \& Jenkins (2015). Here, for the sake of brevity, only the numerical simulations performed by Chialvo et al. (2012) on steady, homogeneous shear flows of spheres (micro-parameters reported in Table 1) are compared with the model results (figure 7), in terms of (a) $\tau^{*}$ versus $\dot{\gamma}^{*}$ for different imposed concentrations, and (b) apparent viscosity $\tau^{*} / \dot{\gamma}^{*}$ versus $\nu$ for different dimensionless shear rates. The constitutive parameters employed in the theoretical model are listed in Table 1. The predictions satisfactorily fit the numerical data in both the fluid-like and solid-like regimes, for $\dot{\gamma}^{*}<10^{-2}$. At large shear rates $\left(\dot{\gamma}^{*}>10^{-2}\right.$, figure $\left.7(\mathrm{a})\right)$, the theory over predicts the numerical data, and, in particular, does not reproduce the dependence of $\tau^{*}$ on $\dot{\gamma}^{* 1 / 2}$, already commented with reference to figure 1 . Nevertheless, this range of non-dimensional shear rate values corresponds to unrealistic values of dimensional shear rates for rigid particles, as soil grains are.

In order to test the theoretical model under saturated conditions, its predictions are compared with the DEM numerical data obtained by Ness \& Sun (2015), described in the previous section. In figure $8(\mathrm{a})$, model predictions are plotted together with the DEM results in terms of $\tau^{*}$ versus $\dot{\gamma}^{*}$ for different values of concentration (as in figure 1(b)). The constitutive parameters employed by the authors are listed in Table 1.

From both qualitative and quantitative points of view, the theoretical predictions are in good agreement with the DEM results. The model correctly describes the transition between 


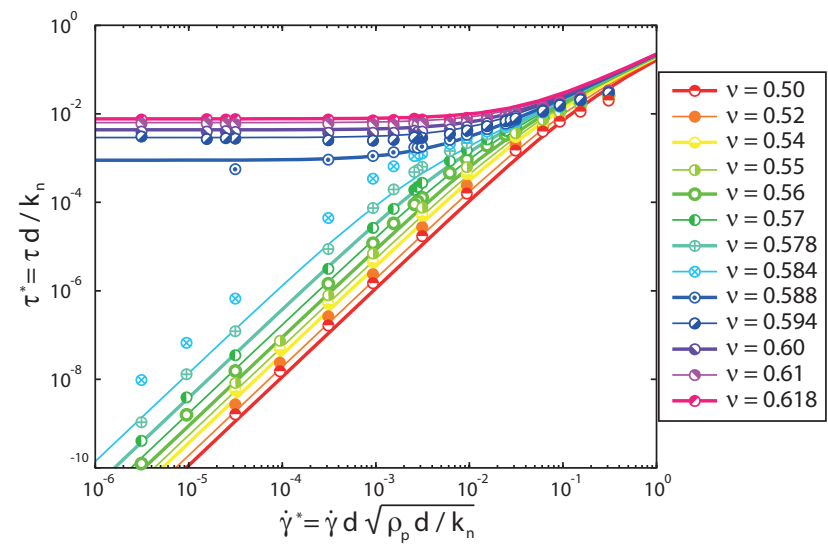

(a)

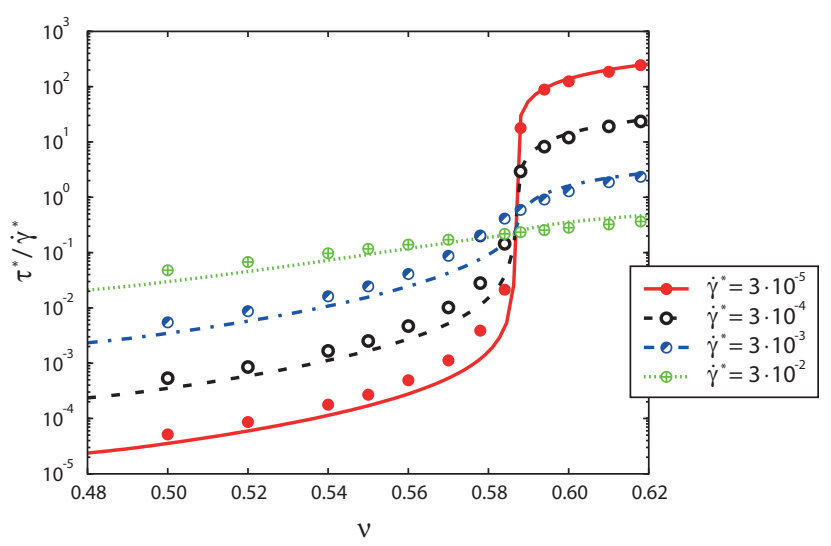

(b)

Fig. 7. Comparison between numerical data (symbols, Chialvo et al., 2012) concerning dry granular flows and model predictions (lines). (a) Dimensionless shear stress versus dimensionless shear rate for different values of $\nu$. (b) Apparent viscosity versus $\nu$ for different $\dot{\gamma}^{*}$ values

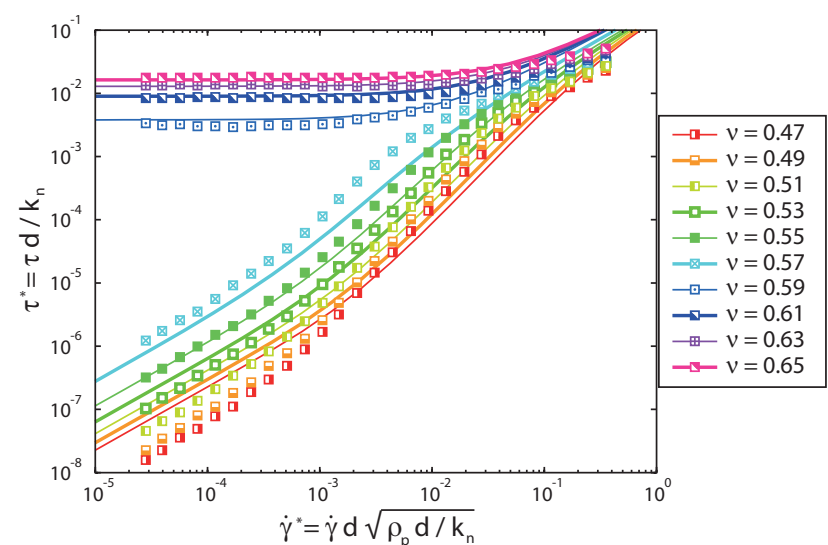

(a)

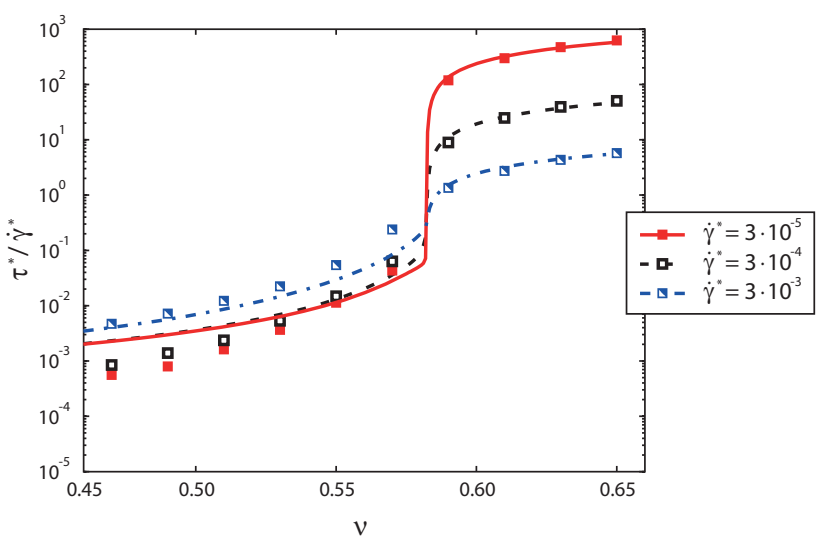

(b)

Fig. 8. Comparison between numerical data (symbols, Ness \& Sun, 2015) concerning saturated granular flows and model predictions (lines). (a) Dimensionless shear stress versus dimensionless shear rate for different values of $\nu$. (b) Apparent viscosity versus $\nu$ for different $\dot{\gamma}^{*}$ values

the different macro-responses mentioned in the previous section. In particular, for $\nu<\nu_{c}$ the model is capable of capturing the transition from Newtonian to Bagnoldian regime. Nevertheless, by comparing numerical data and model predictions, some discrepancies appear evident. For $\dot{\gamma}^{*}>10^{-1}$ the model overestimates the shear stresses, whereas, for $\dot{\gamma}^{*}<$ $10^{-3}$, when the concentration is sufficiently small, the model predicts a less pronounced influence of the concentration on $\tau^{*}$. As far as the first item is concerned, the authors observed that the same discrepancy is evident even under dry conditions (figure 7(a)) and it is a consequence of the limitation of the original constitutive model. As far as the latter one is concerned, the authors think that the numerical data of Ness \& Sun (2015) for these values of $\dot{\gamma}$ are not reliable, since in the numerical analyses the liquid is absent and its effect is taken into account only by introducing equivalent lubrication forces. According to the authors, this approach cannot realistically reproduce the response of the system where the liquid governs the material behaviour, i.e. for sufficiently small values of $\nu$ and $\dot{\gamma}$.
In figure $8(\mathrm{~b})$, for three values of $\dot{\gamma}^{*}$ the comparison between model predictions and the numerical data of Ness \& Sun (2015) is done in terms of apparent viscosity $\tau^{*} / \dot{\gamma}^{*}$ versus solid concentration. The model is capable of capturing the peculiar response of the material for values of concentration very close to $\nu_{c}$. Nevertheless, even in this figure the above mentioned discrepancy is evident for $\nu<0.5$.

\section{Critical discussion}

In this section, the theoretical model is numerically solved in order to illustrate some of its features. The material parameters adopted for the granular phase are listed in Table 1 and refer to an ideal soil ( $\left.d=1 \mathrm{~mm}, \rho_{p}=2500 \mathrm{~kg} / \mathrm{m}^{3}, E_{p}=400 \mathrm{MPa}\right)$ saturated with water $\left(\eta_{0}=10^{-3} \mathrm{~Pa} \cdot \mathrm{s}\right)$.

In figure 9, the dimensionless granular temperature $T /\left(d^{2} \dot{\gamma}^{2}\right)$ predicted by the theoretical model is plotted against concentration, for both dry (dotted lines) and saturated (solid, dot-dashed and dashed lines) granular materials. Under dry conditions, $T$ is proportional to the square of $\dot{\gamma}$, in fact all the lines at different shear rates are superimposed, and, when 
$\nu>\nu_{c}, T$ is not affected by the concentration. If the granular system is saturated, $T$ does not scale quadratically with $\dot{\gamma}$. At low densities $\left(\nu<\nu_{c}\right)$ and low shear rates $\left(\dot{\gamma}^{*}<10^{-5}\right)$, the granular temperature is smaller than in the case of dry systems, and the difference increases for decreasing shear rates. The presence of the viscous dissipation makes saturated systems less agitated with respect to the dry ones, at the same shearing velocity. On the other hand, at large concentrations or large shear rates, the influence of the viscous dissipation can be neglected, so that saturated and dry responses coincide. This implicitly suggests that the system is dominated by the interactions among grains. In particular, when $\nu>\nu_{c}$, the presence of force chains dominate the response of the system, whereas when $\dot{\gamma}^{*}>10^{-5}$, the granular temperature is sufficiently high and the particles are so agitated that collisions become so frequent that their dissipating effect results predominant with respect to the viscous one.

The effect of the liquid phase is reflected on the shear stress, whose trend is plotted in figure 10(a). As for $T$, the shear stress is not affected by the presence of the liquid phase at large concentrations $\left(\nu>\nu_{c}\right)$ or large shear rates $\left(\dot{\gamma}^{*}>10^{-5}\right)$. The differences between dry and saturated responses become evident in the fluid-like regime $\left(\nu<\nu_{c}\right)$, at small shear rates, in the so called Newtonian regime previously introduced. Under such conditions, the shear stress predicted in saturated granular flows is larger with respect to the dry case. In figure 10(b), the granular (dash-dotted line) and the liquid (dashed line) contributions to the shear stress are illustrated for $\dot{\gamma}^{*}=10^{-6}$. At $\nu<\nu_{c}$, the granular phase contribution reduces to the collisional part, $\tau_{g}=\tau_{\text {col }}$, since the quasi-static term vanishes. $\tau_{g}$ is smaller than under dry conditions (dotted line), since the granular temperature is reduced by the presence of the liquid (figure 9). The agitation of grains is damped by the liquid phase, and this is reproduced in the model by means of the energy transferred by the granular to the liquid phase, $\Lambda$, appearing in the granular energy balance. On the other hand, the liquid phase contribution, $\tau_{l}$, is responsible for the increase in the shear stress, and dominates the global response of the mixture at $\nu<\nu_{c}$. When a contact network develops within the medium, i.e. for $\nu>\nu_{c}$, both the granular temperature and the liquid phase do not affect the behaviour of the mixture, which is completely governed by the quasi-static contribution of the granular phase, that is, in this regime the previously mentioned effective stress principle holds.

Figure 11 highlights the role played by the liquid molecular viscosity on the shear stress of the mixture. As was already pointed out, the behaviour of the system is not influenced by the liquid phase, and consequently by its viscosity, in the solidlike regime $\left(\nu>\nu_{c}\right)$. This is evident in figure 11(b), where $\tau^{*}$ is plotted against $\nu$ for different values of molecular viscosity, in a system sheared at $\dot{\gamma}^{*}=10^{-6}$. In figure 11(a), the mechanical behaviour of a fluid-like system at small concentration $(\nu=$ $0.58)$ is illustrated in terms of $\tau^{*}$ versus $\dot{\gamma}^{*}$, for different $\eta_{0}$.

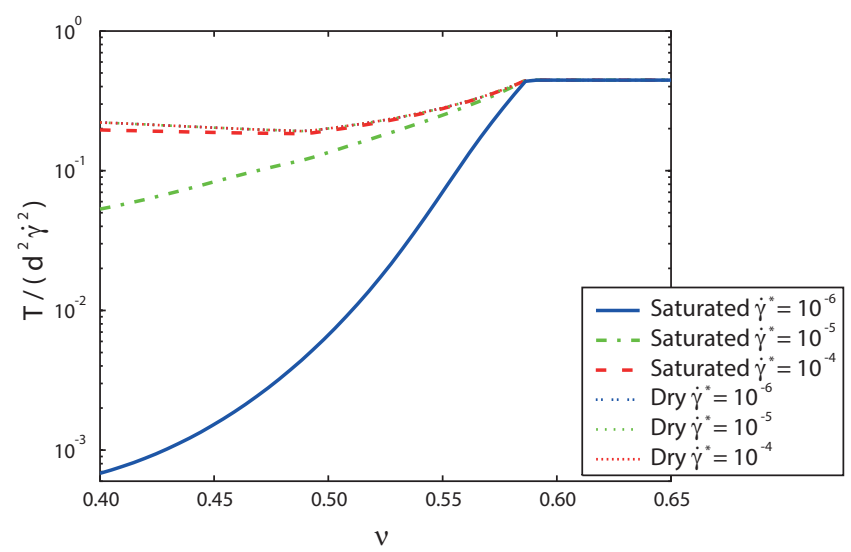

Fig. 9. Dependence of granular temperature on concentration for different $\dot{\gamma}^{*}$ values (dry - dotted lines, saturated - solid, dot-dashed and dashed lines)

As was expected, by increasing $\eta_{0}$, the Newtonian regime $\left(\tau^{*} \sim \dot{\gamma}^{*}\right)$, governed by the liquid viscosity, spans over a wider range of shear rates and the global shear stress increases at small $\dot{\gamma}^{*}$. For low values of $\eta_{0}$ (the dry case can be interpreted as a saturated case for nullifying $\eta_{0}$ ), the behaviour of the saturated system deviates from the dry case (dotted line) only at very small $\dot{\gamma}^{*}$, since the granular phase mainly dominates the material response.

\section{CONCLUSIONS}

The authors have analysed numerical and experimental results concerning either dry and saturated granular flows under steady, simple shear conditions, for different values of both shear rate and concentration. A new constitutive model has been introduced on the base of the mixture theory, according to which the granular and liquid phases are considered separately. The constitutive relationship proposed by the authors: (i) refers to the Representative Elementary Volume and assumes the mean values of all kinematic variables, of both granular and liquid phase, to coincide; (ii) assumes the two phases to work in parallel, as it is according to the well known effective stress principle in case of quasi-static conditions.

As far as the granular, solid fraction is concerned, the authors employ an already conceived constitutive approach capable of describing, at the same time, the mechanical behaviour of agitated granular flows (fluid-like regime) and solid-like granular systems. The approach followed by the authors is based on the introduction of an additional state variable, the granular temperature, describing the system agitation and on the assumption that the well known critical state for highly concentrated granular materials is the limit of steady state conditions for shear strain rates going to zero. Under dry conditions, the dissipation of energy may be due either to frictional contacts belonging to force chains, which can develop only if the concentration is larger than a critical value, or to instantaneous collisions, these latter dominating in case of small values of concentration and large values of shear rate. 


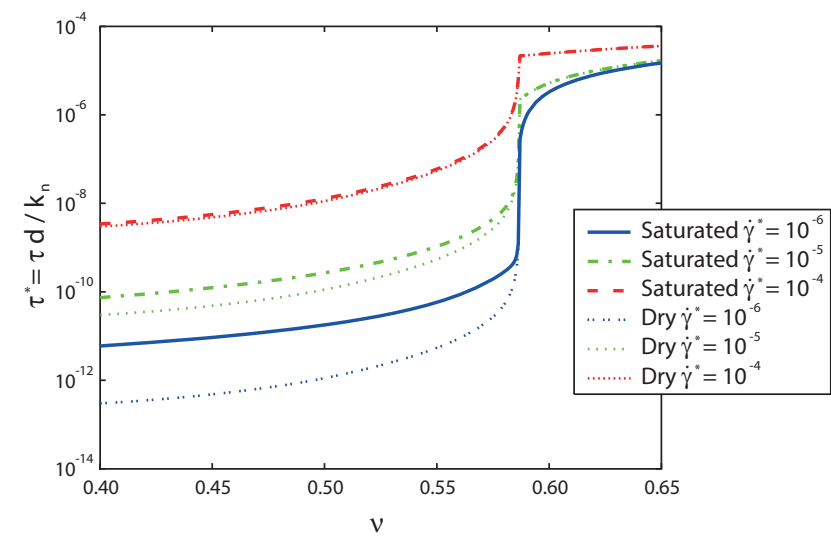

(a)

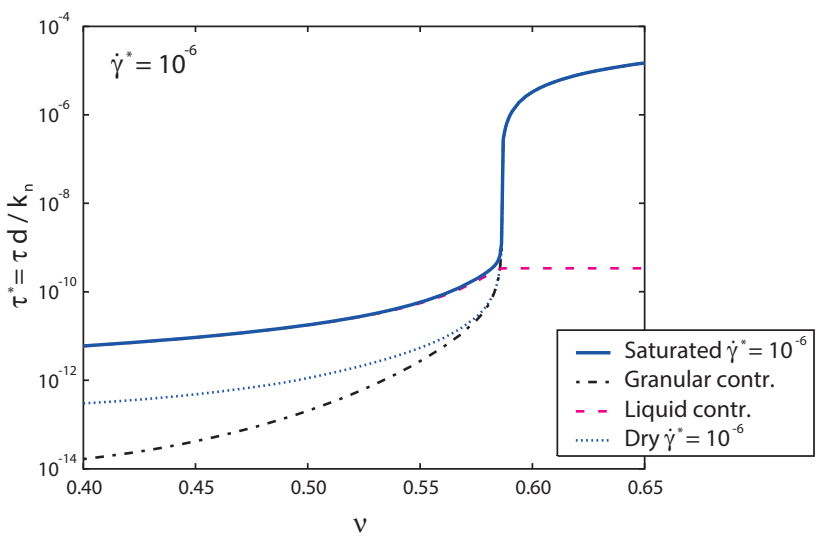

(b)

Fig. 10. (a) Dimensionless shear stress as a function of $\nu$, for different $\dot{\gamma}^{*}$ values, in the case of dry (dotted lines) and saturated (solid, dot-dashed and dashed lines) granular systems. (b) Granular (dash-dotted line) and liquid (dashed line) contributions to the shear stress under saturated conditions (solid line) compared with the shear stress under dry conditions (dotted line)

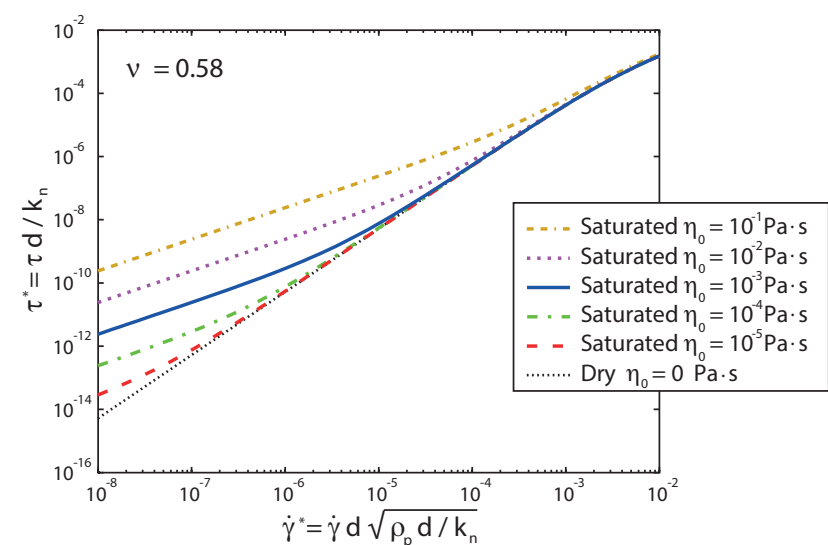

(a)

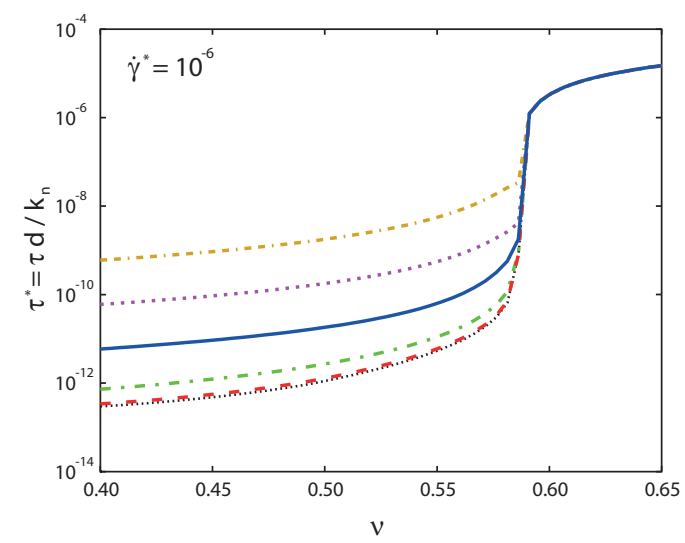

(b)

Fig. 11. Influence of the liquid molecular viscosity, $\eta_{0}$, on the dependence of $\tau^{*}$ on $\dot{\gamma}^{*}$ (a) and $\nu$ (b)

Under saturated conditions, additional dissipation contributions arise in the liquid phase. These are associated with the following effects: (i) deviation of the liquid streamlines due to the presence of particles, behaving as obstacles; (ii) lubrication; (iii) liquid velocity fluctuations due to grains agitation. These dissipation contributions involve the liquid phase and are taken into consideration to define its constitutive relationship by introducing a phase viscosity depending on the concentration according to a suitably calibrated function and on the molecular viscosity of the liquid. On the other hand, the presence of water plays a damping role in reducing the fluctuating energy of the granular phase. The energy balance has thus been used by the authors to describe the coupling. In fact, the granular temperature results to be influenced by the liquid phase molecular viscosity.

The model requires the calibration of only two macroparameters. The first one influencing the critical state line slope, which governs the material response in the solid-like regime. The second one governing the coupling between the granular and the liquid phases, which affects the change in the liquid viscosity for high values of concentration.

The model has been validated against numerical results, and critically discussed. The proposed framework seems to be capable of correctly reproducing the reality by capturing: (i) for sufficiently small values of concentration, the transition from a Newtonian to a Bagnoldian regime, when the shear rate is increased; (ii) the independence of the shear mechanical behaviour of the mixture when the concentration is sufficiently high and the material behaves like a solid.

\section{ACKNOWLEDGEMENTS}

This work has been supported by Fondazione Cariplo, grant $n$. 2016-0769.

\section{APPENDIX: EXPRESSIONS FOR THE GRANULAR CONTRIBUTION}

In Tables 2 and $3, e_{n}$ is the normal coefficient of restitution of the particles. It is a micro-mechanical parameter defined as the ratio of pre-collisional to post-collisional relative velocity between colliding particles in the normal impact direction. Moreover, $\epsilon$ is an effective coefficient of restitution which accounts for the rotation and contact friction of the particles. Its expression has been proposed by Chialvo \& Sundaresan 
NOTATION

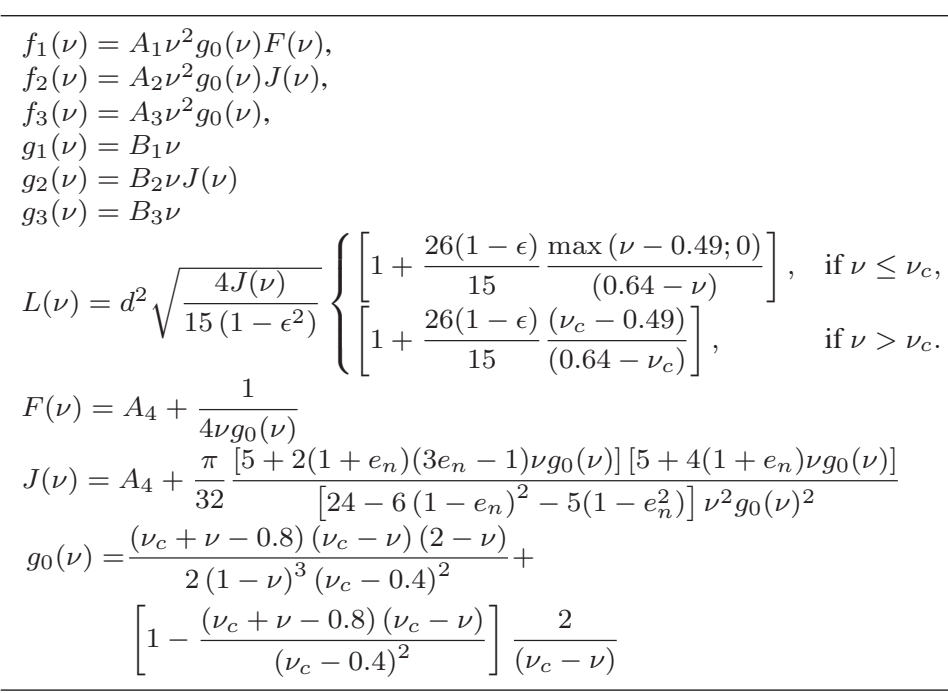

Table 3. List of material parameters for the granular contribution

$$
\begin{aligned}
& A_{1}=4 \rho_{p} \\
& A_{2}=\frac{8}{5 \sqrt{\pi}} \rho_{p} d \\
& A_{3}=\frac{12}{\sqrt{\pi}}\left(1-\epsilon^{2}\right) \rho_{p} \\
& A_{4}=\frac{\left(1+e_{n}\right)}{2} \\
& B_{1}=\frac{5}{12} \sqrt{\pi}\left(1+e_{n}\right)\left(E_{p} \rho_{p}\right)^{1 / 2} \\
& B_{2}=\frac{d}{3}\left(E_{p} \rho_{p}\right)^{1 / 2} \\
& B_{3}=\frac{5}{2}\left(1-\epsilon^{2}\right)\left(E_{p} \rho_{p}\right)^{1 / 2}
\end{aligned}
$$

(2013), on the base of numerical simulations, as a function of the coefficient of normal restitution and the inter-particle friction coefficient $\mu$ :

$$
\epsilon=e_{n}-\mu e^{-3 / 2 \mu} \text {. }
$$

Finally, in the model proposed by Berzi \& Jenkins (2015), $\phi_{s s}^{\prime}$ is evaluated as a function of the concentration, the particle diameter, the shear rate and the granular temperature, derived using discrete numerical simulations of unsteady, homogeneous shearing at large concentration (Sun \& Sundaresan, 2011; Berzi \& Vescovi, 2015). The expression of $\tan \phi_{s s}^{\prime}$ is

$$
\tan \phi_{s s}^{\prime}=\frac{4 J}{5 \sqrt{\pi}\left(1+e_{n}\right)} \frac{d \dot{\gamma}}{T^{1 / 2}}
$$

$a_{0}, C_{\eta} \quad$ dimensionless material parameters

B buoyancy force

$d$ particle diameter

$E_{p} \quad$ particle Young modulus

$e_{n} \quad$ coefficient of normal restitution

$F_{i}{ }^{D} \quad$ Drag force on the $i$-th particle

$F_{j=0,1,2,3} \quad$ functions in the collisional contribution

$F_{\eta} \quad$ viscosity function

$f_{j}, g_{j}, J, f_{\eta} \quad$ auxiliary functions

$k_{n} \quad$ particle elastic stiffness

$L \quad$ correlation length

$n$ number of particles per unit volume

q energy flux

$T$ granular temperature

$t$ time

$\mathbf{u}_{g}, \mathbf{u}_{l} \quad$ local velocity of the granular/liquid phase

$\mathbf{v}_{p} \quad$ single-particle velocity

$\mathbf{v}_{p, i} \quad i$-th particle velocity

$\chi_{g}, \chi_{l} \quad$ granular/liquid internal energy

$\dot{\varepsilon} \quad$ strain rate tensor

$\phi_{s s}^{\prime} \quad$ internal friction angle under simple shear conditions

$\Gamma \quad$ system dissipated energy

$\Gamma_{g}, \Gamma_{l} \quad$ granular/liquid dissipated energy

$\Gamma_{l}^{E} \quad$ liquid dissipated energy due to the presence of grains

$\Gamma_{l}^{g l} \quad$ liquid dissipated energy due to the grains agitation

$\Gamma_{l}^{L} \quad$ liquid dissipated energy due to lubrication effects

$\Gamma_{\mathrm{qs}}, \Gamma_{\mathrm{col}} \quad$ quasi-static/collisional dissipated energy

$\dot{\gamma}$ shear rate

$\dot{\gamma}^{*} \quad$ dimensionless shear rate

$\eta \quad$ liquid viscosity

$\eta_{0} \quad$ molecular viscosity of the liquid

$\Lambda$ energy transferred by the granular to the liquid phase

$\mu$ inter-particle friction coefficient

$\nu$ concentration

$\nu_{c} \quad$ critical concentration

$\rho_{g}, \rho_{l} \quad$ density of the granular/liquid phase

$\rho_{p} \quad$ particle density

$\sigma \quad$ stress tensor

$\sigma \quad$ normal stress

$\sigma_{g}, \sigma_{l} \quad$ granular/liquid normal stress

$\sigma_{\mathrm{qs}}, \sigma_{\mathrm{col}} \quad$ quasi-static/collisional normal stress

$\tau$ shear stress

$\tau^{*} \quad$ dimensionless shear stress

$\tau_{g}, \tau_{l} \quad$ granular/liquid shear stress

$\tau_{\mathrm{qs}}, \tau_{\mathrm{col}} \quad$ quasi-static/collisional shear stress

\section{REFERENCES}

Babic, M., Shen, H. \& Shen, H. (1990). The stress tensor in granular shear flows of uniform, deformable disks at high solids concentrations. J. Fluid Mech. 219, 81-118.

Bagnold, R. (1954). Experiments on a gravity-free dispersion of large solid spheres in a newtonian fluid under shear. Proc. Royal Society A 255, No. 1160, 49-63.

Ball, R. \& Melrose, J. (1997). A simulation technique for many spheres in quasi-static motion under frame-invariant pair drag and brownian forces. Physica A: Statistical Mechanics and its Applications 247, No. 1, 444-472.

Berzi, D., di Prisco, C. \& Vescovi, D. (2011). Constitutive relations for steady, dense granular flows. Phys. Rev. E 84, No. 3, 031301. 
Berzi, D. \& Jenkins, J. (2015). Steady shearing flows of deformable, inelastic spheres. Soft Matter 11, No. 24, 4799-4808.

Berzi, D. \& Vescovi, D. (2015). Different singularities in the functions of extended kinetic theory at the origin of the yield stress in granular flows. Phys. Fluids 27, No. 1, 013302.

Boyer, F., Guazzelli, E. \& Pouliquen, O. (2011). Unifying suspension and granular rheology. Phys. Rev. Lett. 107, 188301.

Campbell, C. (1990). Rapid granular flows. Annu. Rev. Fluid Mech. 22, 57-92.

Campbell, C. (2002). Granular shear flows at the elastic limit. J. Fluid Mech. 465, 261-291.

Chang, C. \& Powell, R. (1994). Effect of particle size distributions on the rheology of concentrated bimodal suspensions. Journal of Rheology 38, No. 1, 85-98.

Chialvo, S., Sun, J. \& Sundaresan, S. (2012). Bridging the rheology of granular flows in three regimes. Phys. Rev. E 85, No. 2, 021305.

Chialvo, S. \& Sundaresan, S. (2013). A modified kinetic theory for frictional granular flows in dense and dilute regimes. Phys. Fluids 25, No. 7, 070603.

Clavaud, C., Bérut, A., Metzger, B. \& Forterre, Y. (2017). Revealing the frictional transition in shear-thickening suspensions. Proceedings of the National Academy of Sciences 114, No. 20, 5147-5152.

Dinkgreve, M., Paredes, J., Michels, M. \& Bonn, D. (2015). Universal rescaling of flow curves for yield-stress fluids close to jamming. Phys. Rev. E 92, No. 1, 012305.

Drew, D. (1983). Mathematical modeling of two-phase flow. Annual Review of Fluid Mechanics 15, No. 1, 261-291.

Eilers, H. (1941). Die viskosität von emulsionen hochviskoser stoffe als funktion der konzentration. Kolloid-Zeitschrift 97, No. 3, 313321.

Einstein, A. (1905). Über die von der molekularkinetischen theorie der wärme geforderte bewegung von in ruhenden flüssigkeiten suspendierten teilchen. Annalen der Physik 322, No. 8, 549-560.

Fall, A., Lemaître, A., Bertrand, F., Bonn, D. \& Ovarlez, G. (2010). Shear thickening and migration in granular suspensions. Phys. Rev. Lett. 105, 268303.

Fernandez, N., Mani, R., Rinaldi, D., Kadau, D., Mosquet, M., Lombois-Burger, H., Cayer-Barrioz, J., Herrmann, H., Spencer, N. \& Isa, L. (2013). Microscopic mechanism for shear thickening of non-brownian suspensions. Phys. Rev. Lett. 111, 108301.

Garzó, V. \& Dufty, J. (1999). Dense fluid transport for inelastic hard spheres. Phys. Rev. E 59, No. 5, 5895-5911.

Goldhirsch, I. (2003). Rapid granular flows. Annu. Rev. Fluid Mech. 35, 267-293.

Hatano, T. (2008). Scaling properties of granular rheology near the jamming transition. J. Phys. Soc. Jpn. 77, No. 12, 123002.

Huang, N., Ovarlez, G., Bertrand, F., Rodts, S., Coussot, P. \& Bonn, D. (2005). Flow of wet granular materials. Phys. Rev. Lett. 94, 028301.

Jackson, R. (2001). The dynamics of fluidized particles. Measurement Science and Technology 12, No. 6, 755.

Jenkins, J. \& Savage, S. (1983). A theory for the rapid flow of identical, smooth, nearly elastic, spherical particles. J. Fluid Mech. 130, 187202.

Ji, S. \& Shen, H. (2006). Characteristics of temporalspatial parameters in quasisolid-fluid phase transition of granular materials. Chin. Sci. Bull. 51, No. 6, 646-654.

Ji, S. \& Shen, H. (2008). Internal parameters and regime map for soft polydispersed granular materials. J. Rheology $\mathbf{5 2}$, No. 1, 87-103.
Kim, S. \& Karrila, S. (1991). Butterworth-heinemann. In Microhydrodynamics, Butterworth-Heinemann.

Krieger, I. M. \& Dougherty, T. J. (1959). A mechanism for non newtonian flow in suspensions of rigid spheres. Transactions of the Society of Rheology 3, No. 1, 137-152.

Kumar, N., Imole, O. I., Magnanimo, V. \& Luding, S. (2014). Effects of polydispersity on the micro-macro behavior of granular assemblies under different deformation paths. Particuology 12, 64-79.

Lin, N., Guy, B., Hermes, M., Ness, C., Sun, J., Poon, W. \& Cohen, I. (2015). Hydrodynamic and Contact Contributions to Continuous Shear Thickening in Colloidal Suspensions. Phys. Rev. Lett. 115, No. 22, 1-5.

Luding, S. (2016). Granular matter: So much for the jamming point. Nature Phys. 12, No. 6, 531-532.

Lun, C. (1991). Kinetic theory for granular flow of dense, slightly inelastic, slightly rough spheres. J. Fluid Mech. 223, 539-559.

Mari, R., Seto, R., Morris, J. \& Denn, M. (2015). Nonmonotonic flow curves of shear thickening suspensions. Phys. Rev. E 91, 052302.

Muir Wood, D. (1990). Soil behaviour and critical state soil mechanics. Cambridge, U.K.: Cambridge University Press.

Ness, C. \& Sun, J. (2015). Flow regime transitions in dense nonbrownian suspensions: Rheology, microstructural characterization, and constitutive modeling. Phys. Rev. E 91, No. 1, 012201.

Ogarko, V. \& Luding, S. (2013). Prediction of polydisperse hardsphere mixture behavior using tridisperse systems. Soft Matter $\mathbf{9}$, No. 40, 9530-9534.

Otsuki, M. \& Hayakawa, H. (2009). Critical behaviors of sheared frictionless granular materials near the jamming transition. Phys. Rev. E 80, No. 1, 011308.

Pan, Z., de Cagny, H., Weber, B. \& Bonn, D. (2015). S-shaped flow curves of shear thickening suspensions: Direct observation of frictional rheology. Phys. Rev. E 92, 032202.

Paredes, J., Michels, M. \& Bonn, D. (2013). Rheology across the zero-temperature jamming transition. Phys. Rev. Lett. 111, No. 1, 015701.

Pitman, E. \& Le, L. (2005). A two-fluid model for avalanche and debris flows. Philosophical Transactions of the Royal Society of London A: Mathematical, Physical and Engineering Sciences 363, No. 1832, 1573-1601.

Redaelli, I., di Prisco, C. \& Vescovi, D. (2016). A visco-elasto-plastic model for granular materials under simple shear conditions. Int. J. Numer. Anal. Methods Geomech. 40, No. 1, 80-104.

Roscoe, K., Schofield, A. \& Wroth, C. (1958). On the yielding of soils. Géotechnique 8, 22-53.

Roscoe, R. (1952). The viscosity of suspensions of rigid spheres. British Journal of Applied Physics 3, No. 8, 267.

Schofield, A. \& Wroth, C. (1968). Critical state soil mechanics. London, U.K.: McGraw-Hill.

Seto, R., Mari, R., Morris, J. \& Denn, M. (2013). Discontinuous shear thickening of frictional hard-sphere suspensions. Phys. Rev. Lett. 111, 218301.

Singh, A., Mari, R., Denn, M. \& Morris, J. (2018). A constitutive model for simple shear of dense frictional suspensions. J. Rheology 62, No. 2, 457-468.

Stachowiak, G. \& Batchelor, A. (2013). Engineering tribology. Oxford, U.K.: Butterworth-Heinemann.

Stokes, G. (1851). On the effect of the internal friction of fluids on the motion of pendulums. Transactions of the Cambridge Philosophical Society 9. 
Sun, J. \& Sundaresan, S. (2011). A constitutive model with microstructure evolution for flow of rate-independent granular materials. J. Fluid Mech. 682, 590-616.

Terzaghi, K. (1943). Theoretical soil mechanics. New York, USA: J. Wiley and Sons.

Verdugo, R. \& Ishihara, K. (1996). The steady state of sandy soils. Soils and Foundations 36, No. 2, 81-91.

Vescovi, D., Berzi, D. \& di Prisco, C. (2018). Fluid-solid transition in unsteady, homogeneous, granular shear flows. Granular Matter 20 , No. 2.

Vescovi, D., di Prisco, C. \& Berzi, D. (2013). From solid to granular gases: the steady state for granular materials. Int. J. Numer. Anal. Methods Geomech. 37, No. 17, 2937-2951.

Vescovi, D. \& Luding, S. (2016). Merging fluid and solid granular behavior. Soft Matter 12, 8616-8628. 